\title{
Critical Questions Regarding Gadolinium Deposition in the Brain and Body After Injections of the Gadolinium-Based Contrast Agents, Safety, and Clinical Recommendations in Consideration of the EMA's Pharmacovigilance and Risk Assessment Committee Recommendation for Suspension of the Marketing Authorizations for 4 Linear Agents
}

\author{
Val M. Runge, $M D$
}

\begin{abstract}
For magnetic resonance, the established class of intravenous contrast media is the gadolinium-based contrast agents. In the 3 decades since initial approval, these have proven in general to be very safe for human administration. However, in 2006, a devastating late adverse reaction to administration of the less stable gadolinium-based contrast agents was identified, nephrogenic systemic fibrosis. The result of actions taken by the European Medicines Agency and the US Food and Drug Administration, stratifying the agents by risk and contraindicating specific agents in severe renal dysfunction, has led to no new cases being identified in North America or Europe. Subsequently, in 2014, long-term deposition in the brain of gadolinium was first shown, after administration of 2 nonionic linear chelates, gadodiamide, and gadopentetate dimeglumine. This has led to an intense focus on the question of in vivo distribution, possible dechelation, and subsequent deposition of gadolinium, together with substantial clarification of the phenomenon as well as stratification of the agents on this basis. This review focuses on 8 critical questions regarding gadolinium deposition in the brain and body, with the answers and discussion therein important for future regulatory decisions and clinical practice. It is now clear that dechelation of gadolinium occurs in vivo with the linear agents and is responsible for this phenomenon, with key experts in the field recommending, except where there is no suitable alternative, a shift in clinical practice from the linear to macrocyclic agents. In addition, on March 10, 2017, the Pharmacovigilance and Risk Assessment Committee of the European Medicines Agency recommended suspension of the marketing authorization for 4 linear gadolinium contrast agents - specifically Omniscan, Optimark, Magnevist, and MultiHance (gadodiamide, gadoversetamide, gadopentetate dimeglumine, and gadobenate dimeglumine) - for intravenous injection. Cited in the report was convincing evidence of gadolinium deposition in the brain months after injection of these linear agents. Primovist/Eovist (gadoxetic acid disodium) will remain available, being used at a lower dose for liver imaging, because it meets an important diagnostic need. In addition, a formulation of Magnevist for intra-articular injection will remain available because of its very low gadolinium concentration.
\end{abstract}

Key Words: contrast media, magnetic resonance, dentate nucleus, safety, gadolinium, gadolinium-based contrast agents, gadodiamide, gadoversetamide, gadopentetate dimeglumine, gadobenate dimeglumine

Received for publication January 11, 2017; and accepted for publication, after revision, March 13, 2017.

From the University Institute of Diagnostic, Interventional and Pediatric Radiology, Inselspital, University Hospital, University of Bern, Bern, Switzerland.

Conflicts of interest and sources of funding: Partial support was provided as unrestricted educational grants from Guerbet and Bayer.

Correspondence to: Val M. Runge, MD, University Institute of Diagnostic, Interventional and Pediatric Radiology, Freiburgstrasse 10 (INO B 106), Inselspital, 3010 Bern, Switzerland.

Copyright (C) 2017 Wolters Kluwer Health, Inc. All rights reserved.

ISSN: 0020-9996/17/5206-0317

DOI: $10.1097 /$ RLI.0000000000000374
(Invest Radiol 2017;52: 317-323)

T he approved gadolinium-based contrast agents (GBCAs) can be differentiated on the basis of structure (ligand type, specifically macrocyclic vs linear) and charge, as detailed in Table $1 .{ }^{1-13}$ Stability both in in vitro and in vivo, relative to the release of gadolinium (which depends upon both thermodynamic and kinetic stability), has been shown to be markedly superior for the macrocyclic agents when compared with the linear agents. ${ }^{14-17}$ The linear nonionic agents (specifically gadodiamide and gadoversetamide) are also markedly less stable by this measure than the linear ionic ones. ${ }^{18}$ All GBCAs cause, in a small percent of patients, mild adverse reactions, including nausea and hives, with a large number of articles in the scientific literature debating the relative percent of such with the different agents. ${ }^{19}$ Severe anaphylactic reactions can also occur with any of the agents, although these are very rare. ${ }^{20}$

The market for the GBCAs in Europe today is similar in size to that in the United States, with Japan being substantially smaller. The total for all 3 areas, in terms of gross sales, is in the range of half a billion dollars. More than 40 million enhanced magnetic resonance scans are performed worldwide each year. The macrocyclic agents dominate the market, with the highest utilization in Europe where it is more than $80 \%$. Overall, among the major manufacturers, Bayer has the largest market share, whereas GE Healthcare has the smallest. In Europe, specifically, Guerbet has the largest market share, whereas in the United States, this is held by Bayer. The 7 primary agents (Table 1) are approved in all 3 of the major markets, with the exception of gadobenate dimeglumine, which is not approved in Japan (source: Arlington Medical Resources, Inc, https://decisionresourcesgroup.com).

Discussing individual agents, the market share for gadodiamide (GE Healthcare) is substantially lower today than in the pastparticularly in Europe and the United States, presumably due to both the impact of nephrogenic systemic fibrosis (NSF) and the issue of gadolinium deposition in the brain. Its current market share in the United States is thought to be below $10 \%$.

Being a late entrant into the field in the United States, gadoterate meglumine (Guerbet) has currently only a small market share there. This will significantly change when gadoversetamide sites are shifted to gadoterate meglumine, which is anticipated (due to the late 2015 purchase by Guerbet of Mallinckrodt's contrast media business). The market share for gadobenate dimeglumine (Bracco) has risen substantially in the last few years in the United States, with this agent benefiting as a result of the falling market share of gadodiamide. In the early years after its approval in the United States, gadoteridol (Bracco) reached a market share near $25 \%$, falling in subsequent years to low single digits, in part due to the loss of a large distribution contract. The market share today of this macrocyclic agent in the United States, which is rising, is thought to be approximately $5 \%$. 
TABLE 1. Clinically Approved GBCAs in Worldwide Use-Names, Structure, and Occurrence of Dentate Nucleus Hyperintensity

\begin{tabular}{|c|c|c|c|c|c|}
\hline Generic Name* ${ }^{*}$ & Trade Name & Acronym & Structure, Charge & Dentate Nucleus Hyperintensity $\ddagger$ & No Dentate Hyperintensity \\
\hline Gadopentetate dimeglumine & Magnevist & Gd-DTPA & Linear, ionic & Kanda et al, ${ }^{1}$ Radbruch et al ${ }^{2}$ & \\
\hline Gadoterate meglumine & Dotarem & Gd-DOTA & Macrocyclic & & Radbruch et $\mathrm{al}^{2}{ }^{2}$ Eisele et $\mathrm{al}^{3}$ \\
\hline Gadoteridol & ProHance & Gd-HP-DO3A & Macrocyclic & & Kanda et $\mathrm{al}^{4}$ \\
\hline Gadodiamide & Omniscan & Gd-DTPA-BMA & Linear, nonionic & $\begin{array}{l}\text { Kanda et al, }{ }^{1} \text { Errante et al, } \\
\quad \text { McDonald et al, }{ }^{6} \text { Quattrocchi et al }{ }^{7}\end{array}$ & \\
\hline Gadobutrol & Gadovist (Gadavist) & Gd-DO3A-butrol & Macrocyclic & & Radbruch et al, ${ }^{8}$ Cao et al ${ }^{9}$ \\
\hline
\end{tabular}

* Listed in order of initial clinical approval.

† Gadoversetamide (Optimark, Gd-DTPA-BMEA) is not listed, as its use is being substituted by gadoterate meglumine.

$\$$ Primary author listed for confirming report, with reference provided in parentheses.

$\S$ Study limited due to applied dose.

GBCA indicates gadolinium-based contrast agent.

The focus of this review is to discuss gadolinium deposition in the brain and body, ${ }^{21}$ examining 8 key issues, which cover the status of current knowledge, further evaluations that need to be pursued, and the potential impact of these on regulatory approval and use of the GBCAs. It is well established today which of the agents cause hyperintensity of the dentate nucleus (on unenhanced T1-weighted magnetic resonance (MR) scans, after multiple prior administrations), which parallels their in vitro and in vivo stabilities. This is detailed in Table 1, with an example provided in Figure 1, and will not be discussed in detail further.
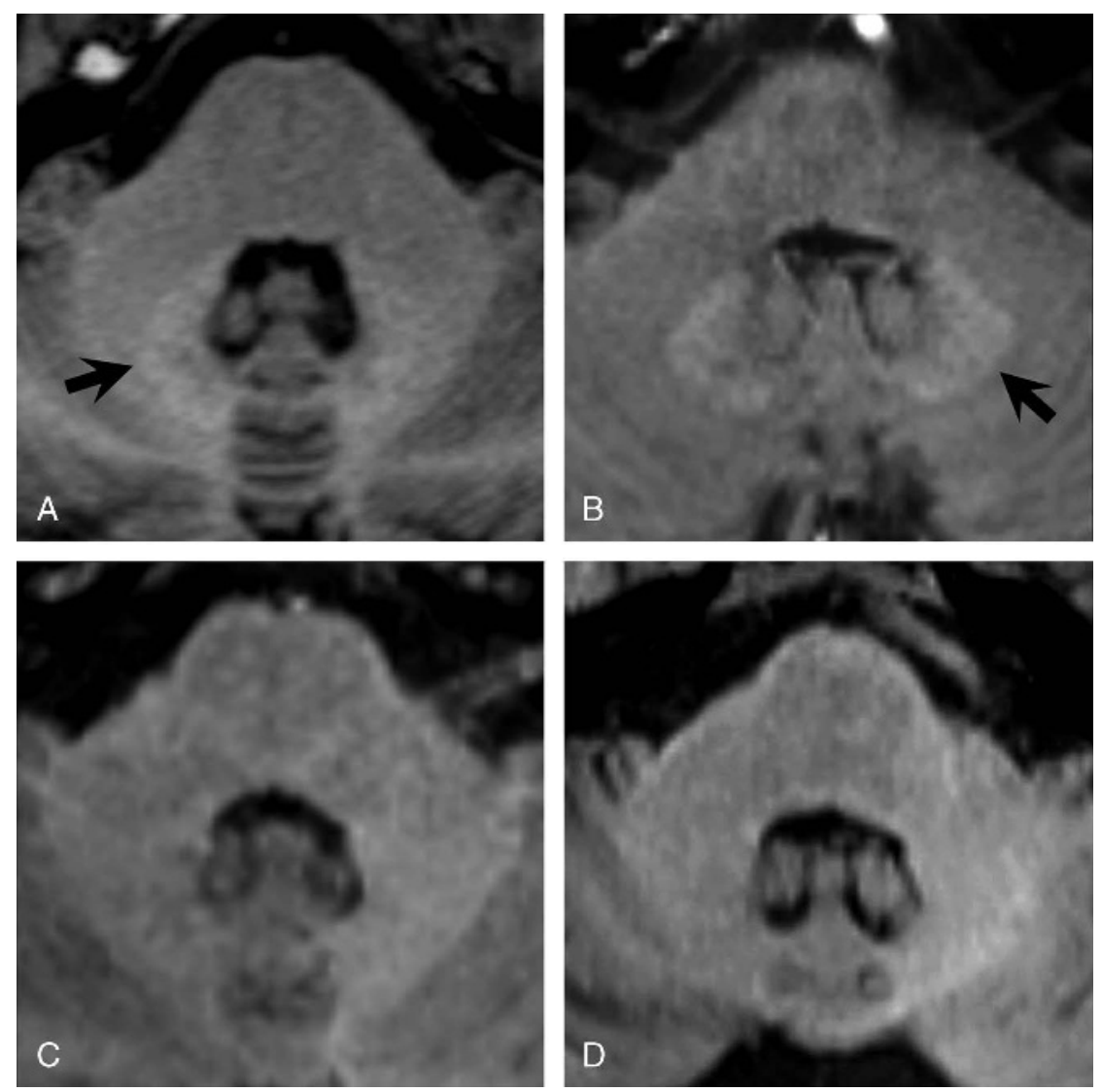

FIGURE 1. Unenhanced axial T1-weighted images through the level of the dentate nucleus in patients having (A) 14 prior injections of gadopentetate dimeglumine, (B) 9 prior injections of gadobenate dimeglumine, (C) 6 prior injections of gadobutrol, and (D) 10 prior injections of gadoterate meglumine. Hyperintensity of the dentate nucleus (arrows) is seen as a residual after multiple injections of ( $A$ and $B$ ) the linear agents gadopentetate dimeglumine and gadobenate dimeglumine, but not after (C and D) the macrocyclic agents gadobutrol and gadoterate meglumine. Baseline images obtained before any contrast administrations (not shown) were normal in all 4 patients. Images courtesy of Alexander Radbruch. 


\section{Clinical Implications of Gadolinium Accumulation in the Brain}

Although there are suggestions in the literature that patient symptoms may be associated with or attributed to the administration of GBCAs, specifically in regard to brain deposition of gadolinium, no definitive such studies have yet been published. Two studies that have drawn substantial attention, specifically the studies of Semelka et $\mathrm{al}^{22}$ and Burke et $\mathrm{al}^{23}$ are based solely on responses to surveys (eliciting symptoms) provided to individuals who believe that they have symptoms (selection bias), and thus do not allow for any scientific conclusions to be drawn. In both, the data were self-reported, unconfirmed medically, and not linked to imaging or histology results. Welk et $\mathrm{al}^{24}$ evaluated retrospectively the possibility that GBCA exposure could cause symptoms of parkinsonism. This is an important area for evaluation, given the involvement of the substantial nigra and the associated signaling to the globus pallidus in that disease. The study used multiple administrative databases and analyzed more than 200,000 patients. The incidence of parkinsonism without and with GBCA administration was comparable. This study, with its inherent limitations, provides some reassurance that gadolinium deposition in the globus pallidus does not lead to neuronal damage, which manifests itself as parkinsonism. However, baseline symptomatology of patients in possible retrospective studies (eg, parkinsonism, brain tumors) is often severe, and subtle cerebellar signs might easily escape detection. Evaluation of pharmacovigilance databases by the 4 major producers of GBCAs worldwide does not reveal reports of any chronic symptoms with sufficient scientific evidence to suggest a causal association. One area that needs specific evaluation, however, is the possible risk of repeated linear GBCA injections in patients receiving drugs that are neurotoxic to the dentate nucleus. Metronidazole, a common antibacterial and antiprotozoal agent, is known to have neurologic toxicity with a predilection for the dentate nucleus, with signal changes on MR imaging seen in the dentate and red nuclei. ${ }^{25}$

\section{Accumulation of Gadolinium in Tissues Other Than the Brain}

Clinical data regarding gadolinium deposition in tissues other than the brain after GBCA administration are limited. An important lesson from the NSF experience is the difficulty in ascertaining with certainty the specific contrast media and number of administrations that any specific patient may have been given. Health care providers have access to data only within their own system, typically, with patients often crossing between providers. Patients also do not reliably know whether an examination was performed with a contrast agent or not, or even whether the examination was MR as opposed to CT. Most certainly patients in general do not know the specific contrast agent used, and there is no definitive medical record in existence that provides this information.

It should be stressed that, to date, the form of gadolinium, other than in the brain, has rarely been investigated. This could be as the intact chelate, or dissociated and soluble (presumably bound to a macromolecule), or precipitated. These will neither behave similarly in the body nor have similar interactions with important structures such as ion channels, which control the flow of ions across the cell membrane.

Care needs also to be exercised, in evaluating tissue deposition of gadolinium, relative to the time interval between the last contrast administration and tissue collection. This is important in any study attempting to differentiate between the different agents, given the temporal elimination of the agents. An example is the study by Murata et al. ${ }^{26}$ Study limitations, acknowledged by the authors, include a lack of control for confounding factors, including specifically prior exposure to other GBCAs, and the varying time between contrast administration and tissue sampling. Looking at the time between the last contrast agent administration and death (tissue sampling), this varied between 5 and
392 days. Such retrospective clinical studies, due to critical confounding factors, should be viewed with caution. These difficulties lead to the conclusion that such fundamental questions are best addressed in preclinical studies, which allow for control of all potential confounders.

Lancelot recently published a meta-analysis from preclinical and clinical pharmacokinetic data concerning gadolinium in blood, urine, and bone. ${ }^{27}$ This work confirmed the existence of prolonged excretion of gadolinium after contrast administration, specifically a residual excretion phase much slower than the conventional elimination phase. This is indirect evidence of a deep compartment of distribution for gadolinium in the body after contrast administration. The slopes of the residual excretion phase highly correlated with thermodynamic stability constants. The data suggest that stability of the GBCA influences the residence time in this deep compartment. Much higher bone clearance rates were determined for the macrocyclic as compared with the linear GBCAs, translating into a much shorter bone half-life. The difference in bone clearance between ${ }^{14} \mathrm{C}$ - and ${ }^{153} \mathrm{Gd}$-labeled agents further supports that macrocyclic agents are cleared by slow washout like any inert small molecule, without biochemical transformation, whereas the linear GBCAs undergo disassociation and deposition. The gadolinium deposited in the bone by a linear agent could later be mobilized and released back into the blood, with bone thus serving as a reservoir. In addition to these findings regarding differences in the clearance of the GBCAs, the quantities of gadolinium measured in the skin, bone, and brain are much lower with the macrocyclic GBCAs. Thus, distinguishing between linear and macrocyclic GBCAs, a difference is noted regarding both the quantity of the gadolinium present in tissues as well as the rate of wash out.

Gadolinium is a lanthanide metal (atomic number 64), 1 of 15 such elements with atomic numbers 57 (lanthanum) to 71 (lutetium). For metals in general, the skeleton is a target organ, with bioaccumulation therein, either for storage of useful elements or protection against toxic ones. ${ }^{28}$ There is limited toxicologic information available regarding accumulation in the body of lanthanides. Of note, Fosrenol (lanthanum carbonate) is commonly prescribed (oral administration) to reduce serum phosphate in end-stage renal disease patients. Possible adverse effects have been investigated in this population, with no evidence of bone toxicity. ${ }^{29}$ Despite progressive accumulation of lanthanum in bone, no clinical symptoms possibly related to toxic effects have been noted. ${ }^{30}$ At this time, no conclusive evidence exists in the scientific literature to show neurotoxicity with other lanthanide elements.

\section{Recent Animal Investigations}

Three early animal investigations were crucial in improving our understanding of gadolinium deposition in the brain. ${ }^{31-33}$ After repeated administration of linear GBCAs (specifically gadobenate dimeglumine, gadopentetate dimeglumine, and gadodiamide), progressive T1 signal hyperintensity in the rat equivalent of the dentate nucleus was demonstrated, with deposition of gadolinium confirmed in the tissue. This was in distinction to the macrocyclic GBCAs (specifically gadoterate meglumine and gadobutrol) for which no effect was observed. This work was performed by 2 of the major 4 manufacturers of GBCAs. Given the lack of published work from the other 2 manufacturers disputing these findings, it is highly likely that this work has been internally confirmed by both.

Several additional important animal studies have been published in the last year, providing further clarification. In renally impaired rats, when compared with rats with normal renal function, gadolinium uptake, after multiple injections of gadodiamide, was potentiated in the cerebellum, brain, and bones. ${ }^{34}$ This study also showed that plasma gadolinium was predominately in a disassociated, soluble form. Total Gd concentration in the brain correlated with renal function. Translating these results to clinical practice, concern is raised due to the substantial population of patients with moderate renal failure. The data in this study 
suggest that moderate renal failure increases the risk of Gd uptake in the brain and other tissues, advocating a special warning for gadodiamide, as well as potentially all the linear agents, for this patient population. Given similarities in stability and potential for dechelation, these results would also be expected with gadoversetamide.

Research from GE Healthcare has further confirmed the dosedependent deposition of gadolinium in the rat brain after gadodiamide administration. ${ }^{35}$ Evaluation of low- and high-dose groups indicated that the uptake mechanisms were not saturated (greater deposition occurred at higher doses). Quantitative measures revealed partial clearance (a decrease by about half) in comparison with the 1- and 20 -week postdose groups. This long-term follow-up (20 weeks in the rat) is comparable to 15 human years. Histologic evaluation of the brain, limited only to hematoxylin-eosin stains, revealed no changes. A subsequent letter to the editor from neurology and neuropathology colleagues, however, stated that normal-appearing hematoxylin-eosin-stained sections would not exclude gadolinium-related neurotoxicity, and that such an assessment should include lactate dehydrogenase immunoreactivity or electron microscopy, in addition to quantitative measures of reactive astrogliosis and microglial activation. ${ }^{36}$

In an additional study, after repeated high dosing in rats with normal renal function, NSF like macroscopic and microscopic lesions in the skin were only seen with gadodiamide. ${ }^{37}$ These were not seen with gadopentetate dimeglumine and the 2 evaluated macrocyclic GBCAs, gadobutrol and gadoteridol. No histologic changes were observed with any agent in the brain. The gadolinium concentration in the skin was determined to be highest with gadodiamide, with the rank being gadodiamide $>>$ gadopentetate dimeglumine $>>$ gadobutrol $=$ gadoteridol, correlating well with the higher kinetic stability of the macrocyclic agents. Gadolinium concentration in the brain ranked similarly for the agents with the exception that gadodiamide $=$ gadopentetate dimeglumine. Laser ablation inductively coupled plasma mass spectrometry revealed high gadolinium concentrations in the deep cerebellar nuclei and the granular layer of the cerebellar cortex with the 2 linear agents, but not with the macrocyclic agents. A similar distribution has recently been confirmed in a single human case report, using laser ablation inductively coupled plasma mass spectrometry, after 4 doses of linear GBCAs. ${ }^{38}$

Further evidence supporting the stability and excretion of macrocyclic agents is provided by juvenile rat (those with immature renal function) toxicologic investigations pursued just before the knowledge emerged concerning gadolinium deposition by linear agents in the brain. ${ }^{39,40}$ The first study evaluated only gadoterate meglumine and involved both single and multiple administrations. Total Gd concentrations were similar in all tissues whether after single or repeated dosing, with only trace amounts of gadolinium quantifiable at the end of the treatment-free period, almost exclusively in the kidneys. In the second study, repeated administrations of gadoteric acid were shown to be well tolerated, despite the immature renal function. In distinction, gadodiamide, which was also evaluated, induced significant mortality (4 of 14 rats) and morbidity. Alopecia, hyperpigmentation, and severe dermal and epidermal lesions were seen after gadodiamide administration. Higher tissue concentrations of gadolinium were also found with gadodiamide as compared with gadoteric acid, and dissociation of gadodiamide was noted to be present in skin and liver.

The most recent animal study assessed and quantified the chemical form of residual gadolinium in the brain after repeated dosing in rats with normal renal function. ${ }^{15}$ For all 3 linear agents evaluatedgadodiamide, gadopentetate dimeglumine, and gadobenate dimegluminethe residual Gd was present in at least 3 distinctive forms - soluble small molecules (likely the intact chelate), soluble macromolecules, and insoluble Gd, with no relevant differences between these agents. The authors hypothesized that the latter 2 forms were most likely responsible for the residual T1 high signal intensity seen in clinical patients in the dentate nucleus and other structures. Gd concentrations in the brain with the macrocyclic agents evaluated - gadobutrol and gadoterate meglumine - were substantially lower, with the Gd only present as soluble small molecules. This work again emphasizes the kinetic inertness of the macrocyclic agents and its crucial importance in prevention of release of Gd in the brain as compared with the linear, kinetically less restricted, GBCAs. The macrocyclic agents seem to be still intact in brain tissue, whereas up to $27 \%$ of the fraction retained in the brain with the linear agents evaluated seems to be present as macromolecules, the result of dechelation.

Not yet well understood is how the GBCAs reach the brain, given that they do not cross the intact blood-brain barrier. However, recent data, not yet replicated in animals, show that intrathecal injection of a GBCA leads to T1 signal hyperintensity of the dentate nucleus and globus pallidus, suggesting entry via the glymphatic system, which is a brain-wide cerebrospinal fluid (CSF)-interstitial fluid mechanism by which interstitial solutes are cleared. ${ }^{41}$ That CSF is a potential pathway of GBCA entry into the brain was confirmed in a recent animal study by Jost et al. ${ }^{42}$ No differences in penetration and distribution into the CSF was noted between the 6 evaluated, marketed GBCAs.

\section{Gadolinium in the Environment}

Gadolinium is today found in nature, as a result of the impact of its use by humans (anthropogenic gadolinium). ${ }^{43}$ It has been reported in rivers, lakes, groundwater, and tap water worldwide. The anthropogenic Gd found is dominated by highly stable, water-soluble complexes, specifically GBCAs administered for MR imaging and subsequently found in waste water (ultimately passing through sewage treatments plants into surface water). ${ }^{44}$ In evaluating the potential for adverse effects with human exposure to gadolinium in tap water, it should be noted that the systemic bioavailability after oral exposure is minimal. There is no evidence to date for substantial systematic exposure of humans to $\mathrm{Gd}$ on the basis of uptake from the environment.

The effects of UV radiation on the GBCAs were recently investigated because these agents would be exposed to UV when present as anthropogenic gadolinium and because of the increasing use of UV irradiation in wastewater treatment processes. Four agents were evaluated. Gadopentetate dimeglumine, gadobutrol, and gadoterate meglumine all showed high stability in irradiation experiments, with gadobenate dimeglumine degrading, yielding by-products and dissociated gadolinium. ${ }^{45}$

\section{Minimizing Risk}

Gadolinium-based contrast agents should only be administered if the information so provided is necessary, and specifically expected to increase the confidence in correct disease diagnosis or assessment thereof, or disease exclusion. No GBCAs are approved for central nervous system (CNS) or body indications (excluding the liver) at lower than standard dose, specifically $0.1 \mathrm{mmol} / \mathrm{kg}$. Indeed, in the case of gadobenate dimeglumine, the application to the Food and Drug Administration (FDA) for approval of half dose $(0.05 \mathrm{mmol} / \mathrm{kg})$ for the CNS was rejected in years past, despite the higher relaxivity, presumably due to a lack of comparable efficacy. Similarly, the attempt to receive approval by the FDA for half dose gadopentetate dimeglumine in young children, potentially reducing the contrast burden in this vulnerable population, failed. Approved dose translates to the recommended lowest effective dose, with health care providers advised not to decrease dose due to the potential lack of efficacy. As with any injection, subsequent repeated injections of a GBCA should only be performed after careful individual patient evaluation of benefit versus risk.

Two patient groups are potentially at higher risk for gadolinium deposition in the brain after administration of GBCAs. Renal impairment leads to longer exposure times to the administered dose, increasing gadolinium deposition in tissue, and specifically in the brain for the linear chelates. Patients with chronic disease may have repeated 
examinations and are, thus, also at potential risk for greater gadolinium deposition. T1-weighted hyperintensity in the dentate nucleus has been shown recently to occur at lower cumulative doses of linear agents in patients on dialysis than in prior studies of patients with normal renal function. ${ }^{46}$ Evidence-from both clinical and preclinical studies- to date favors that patients with impaired renal function are at higher risk for gadolinium accumulation in the brain and in bone, with this conclusion applying only to the group of agents that have such gadolinium deposition, the linear chelates. ${ }^{34}$ Both cited studies furthermore noted deposition of gadolinium in the choroid plexus (beyond what was previously established, specifically the dentate nucleus and the globus pallidus). The Cornell and Columbia groups subsequently published a study of patients receiving 35 or more linear GBCA administrations with normal renal function. Increased signal intensity on unenhanced T1-weighted images, presumed to be due to gadolinium deposition, was much more extensive anatomically than previously described, being seen also in the posterior thalamus, red nucleus, substantia nigra, cerebellar peduncle, and colliculi. ${ }^{47}$

\section{Suitable Alternatives}

No suitable alternatives currently exist for CNS and body MR imaging relative to the use of the extracellular renally excreted GBCAs. The data previously presented support use of the macrocyclic agents as opposed to the linear agents.

Two GBCAs have specific liver approval, and although both are linear agents, the hepatocyte uptake provides in delayed imaging additional information for lesion characterization and detection. These 2 agents differ according to administered dose and the timing of imaging for the hepatocyte selective phase. Consideration of in vitro stability studies, ${ }^{14}$ degree of liver enhancement, quantity of administered gadolinium (1/4 for gadoxetate disodium when compared with the other GBCAs, because it is formulated at $0.25 \mathrm{mmol} / \mathrm{mL}$ and administered at $0.1 \mathrm{~mL} / \mathrm{kg}$ ), and ease of obtaining delayed images (within 10-20 vs 60-90 minutes) all favor 1 agent, gadoxetate disodium, if other alternatives are not available.

\section{Designs for Future Nonclinical Studies}

Further work is needed to identify and characterize the nature of the gadolinium binding macromolecules (with high molecular weight) as well as the insoluble fraction, detected after injection in animals of the linear chelates. It is important to confirm as well that the small soluble molecules detected represent intact GBCA. This work would logically follow that described in the third section of this review describing recent animal investigations.

In-depth histopathology studies are indicated beyond traditional light microscopy and stains. Possible more specific markers include glial fibrillary acidic protein and ionized calcium binding adaptor molecule 1 (a microglia/macrophage-specific calcium-binding protein). Magnetic resonance spectroscopy might also be of value to assess brain metabolism and the possible effect of repeated injections of linear GBCAs in suitable rat models.

The numerous advantages associated with nonclinical models should be acknowledged, for example, their prospective nature, the possibility of euthanasia at study completion with in-depth tissue analysis, and the large number of potential validated end points. Furthermore, rat models are considered very translational with respect to the study of neurologic deficits, specifically including akinesia, tremor, postural deficits, and dyskinesia. ${ }^{48,49}$

Animal investigations would thus be important to assess potential neurologic effects of gadolinium deposition in the brain. Motor coordination function (and thus specifically involvement of the dentate nucleus) can be evaluated with standard tests including balancing on a rotating rod (the rotarod test) and an analysis of gait. Additional established behavioral animal tests that assess partially cognitive function include the open-field test (which assesses motor activity and reactivity to a novel environment), the water maze test (which evaluates learning and memory), grooming analysis, and eye blink conditioning. For 1 macrocyclic agent, gadoterate meglumine, such behavioral tests (specifically the water maze and open field) have been performed in juvenile rats after both single and repeated contrast administrations, and did not show any change. ${ }^{39}$

Intracellular metabolic, neurofunctional, and neuromorphological biomarkers should also be investigated through in vitro and in vivo models. These approaches could potentially detect not only neurotoxic but also neuromodulator effects caused by gadolinium deposition in the brain. Because the brain has permanent active retrocontrol loops, gadolinium deposition could subtly influence some intracellular, synaptic, axonal, neurochemical, or neuroelectrical biomarkers.

\section{Options for Clinical Studies}

There are many challenges for clinical trials, whether prospective, retrospective, or involving data from large registries, to determine if gadolinium deposition in the brain has any clinical relevance or is associated with any adverse health effects. The greatest challenge is that no clear, definitive end points have been identified. One of the most important primary end points would be symptomatology involving a function controlled by the affected basal ganglia, yet, to date, no such correlated symptom has been identified. The basal ganglia involved control many different motor and cognitive processes, which are also involved by many CNS diseases and therapeutic drugs. An additional major challenge is selection of a suitable patient population. Patients are needed who have been or will be subject to many repeat contrast-enhanced MR scans due to their disease process, such as multiple sclerosis or primary brain tumors. Unfortunately, the overlap in potential symptomatology with the primary disease of the patient and drugs used for treatment limits options in these populations. Outside of the CNS, the most obvious population with repeated MR studies is that of patients undergoing screening for breast cancer. Performance of clinical studies in this patient population unfortunately raises ethical questions.

Prerequisites for an ideal study include documentation of a normal baseline, including a thorough neurologic examination with cognitive function testing, an adequate control group, and exclusion of prior GBCA exposure. Using a prospective design, it would unfortunately take many years to obtain reliable information, given the need for multiple exposures to a GBCA over time.

The large electronic available databases do offer the possibility of retrospective studies, and in the "big data" era, it could reveal weak, subsymptomatic, and chronic clinical signs. Retrospective studies and those involving large registries are limited, however, in 2 important ways. First, no baseline neurologic or cognitive testing can be performed. Second, the specific GBCAs administered typically cannot be identified with confidence. It would be important for any such study to identify patients exposed to 1 and only 1 GBCA, avoiding possible carryover effects from previous administration of other agents.

\section{CONCLUSIONS}

An unstated question that is critical to the analysis of the GBCA data, specifically for each of the linear agents, is whether there are patient populations or indications for which there are no suitable alternatives. Clearly, there are alternatives to the majority of these agents. The end result in terms of the actions by the regulatory authorities, and specifically the FDA and European Medicines Agency (EMA), is likely to be based upon a combination of science, politics, and possible legal action.

In the last decade, due to NSF and the evolving knowledge concerning gadolinium deposition in the brain, there has been a dramatic shift in market share for the 4 major manufacturers involved-Bayer, Guerbet, Bracco, and General Electric. These sales figures, however, 
are not publicly available. The market share for both gadodiamide and gadopentetate dimeglumine has fallen substantially, with Bayer substituting gadobutrol (a macrocyclic agent) for gadopentetate dimeglumine (a linear agent). Given Guerbet's acquisition of Mallinckrodt's contrast media business in late 2015, the replacement of gadoversetamide (a linear agent similar in structure to gadodiamide) by gadoterate meglumine (a macrocyclic agent) is anticipated. In addition, in early 2017, at the European Congress of Radiology, GE Healthcare announced that it would launch in Europe an ionic macrocyclic agent, "gadoteric acid." Movements by region of the world in terms of specific contrast agent use have been substantially different, with the greatest changes as well as level of concern by patients and physicians being in Europe. Of the world's regulatory agencies, the EMA has been the most progressive in dealing with this latest issue, similar to how this agency dealt with NSF.

After a year-long consideration of the latest issue (gadolinium deposition in the brain and body), including many meetings, 4 rounds of questions from the EMA to industry with responses, and an ad hoc expert group meeting, the Pharmacovigilance and Risk Assessment Committee concluded it's assessment of the gadolinium agents and, on March 10, 2017, recommended the following regulatory action: Suspension of the marketing authorization of 4 linear GBCAs (gadodiamide, gadoversetamide, gadopentetate dimeglumine, and gadobenate dimeglumine) was advised, which would leave - of the linear agents - only gadoxetic acid on the market for liver scans and a formulation of gadopentetic acid for joint injections. Approval for the macrocyclic agents was left unchanged; this recommendation was sent to the Committee for Medicinal Products for Human Use of the EMA, which-barring action by the companies concerned to request the Pharmacovigilance and Risk Assessment Committee to re-examine its recommendations - will adopt the Agency's opinion at 1 of its meetings in 1 to 2 months. The final stage of review is the adoption by the European Commission of a decision, applicable and legally binding, for all European Union Member States.

Given the concern with gadolinium deposition, use of exclusively the macrocyclic agents is advocated for CNS and body imaging. Two agents exist with hepatobiliary properties, with both linear in nature. This clinical application is important, suggesting that continued approval of 1 -logically that with the lowest gadolinium dose and greatest utility - be maintained. Despite that hepatobiliary agents are less likely to be used in any single patient with an extreme number of doses, development of macrocyclic hepatobiliary agents is also advocated. These were pursued in the past, ${ }^{50,51}$ with clinical approval not sought due to changing market and company dynamics at that time.

\section{REFERENCES}

1. Kanda T, Ishii K, Kawaguchi H, et al. High signal intensity in the dentate nucleus and globus pallidus on unenhanced T1-weighted MR images: relationship with increasing cumulative dose of a gadolinium-based contrast material. Radiology. 2014;270:834-841

2. Radbruch A, Weberling LD, Kieslich PJ, et al. Gadolinium retention in the dentate nucleus and globus pallidus is dependent on the class of contrast agent. Radiology. 2015;275:783-791.

3. Eisele P, Alonso A, Szabo K, et al. Lack of increased signal intensity in the dentate nucleus after repeated administration of a macrocyclic contrast agent in multiple sclerosis: an observational study. Medicine (Baltimore). 2016;95:e4624.

4. Kanda T, Osawa M, Oba $\mathrm{H}$, et al. High signal intensity in dentate nucleus on unenhanced T1-weighted $\mathrm{mr}$ images: association with linear versus macrocyclic gadolinium chelate administration. Radiology. 2015;275:803-809.

5. Errante Y, Cirimele V, Mallio CA, et al. Progressive increase of T1 signal intensity of the dentate nucleus on unenhanced magnetic resonance images is associated with cumulative doses of intravenously administered gadodiamide in patients with normal renal function, suggesting dechelation. Invest Radiol. 2014;49:685-690.

6. McDonald RJ, McDonald JS, Kallmes DF, et al. Intracranial gadolinium deposition after contrast-enhanced MR imaging. Radiology. 2015;275:772-782.

7. Quattrocchi CC, Mallio CA, Errante Y, et al. Gadodiamide and dentate nucleus T1 hyperintensity in patients with meningioma evaluated by multiple follow-up contrast-enhanced magnetic resonance examinations with no systemic interval therapy. Invest Radiol. 2015;50:470-472.

8. Radbruch A, Weberling LD, Kieslich PJ, et al. High-Signal intensity in the dentate nucleus and globus pallidus on unenhanced T1-weighted images: evaluation of the macrocyclic gadolinium-based contrast agent gadobutrol. Invest Radiol. 2015;50 805-810.

9. Cao Y, Huang DQ, Shih G, et al. Signal change in the dentate nucleus on T1weighted MR images after multiple administrations of gadopentetate dimeglumine versus gadobutrol. AJR Am J Roentgenol. 2016;206:414-419.

10. Weberling LD, Kieslich PJ, Kickingereder P, et al. Increased signal intensity in the dentate nucleus on unenhanced $\mathrm{T} 1$-weighted images after gadobenate dimeglumine administration. Invest Radiol. 2015;50:743-748.

11. Ramalho J, Castillo M, AlObaidy M, et al. High signal intensity in globus pallidus and dentate nucleus on unenhanced T1-weighted MR Images: evaluation of two linear gadolinium-based contrast agents. Radiology. 2015;276:836-844.

12. Ichikawa S, Motosugi U, Omiya Y, et al. Contrast agent-induced high signal intensity in dentate nucleus on unenhanced T1-weighted images: comparison of gadodiamide and gadoxetic acid. Invest Radiol. 2017;52.

13. Kahn J, Posch H, Steffen IG, et al. Is there long-term signal intensity increase in the central nervous system on T1-weighted images after MR imaging with the hepatospecific contrast agent gadoxetic acid? A cross-sectional study in 91 patients. Radiology. 2017;282:708-716.

14. Frenzel T, Lengsfeld $P$, Schirmer $H$, et al. Stability of gadolinium-based magnetic resonance imaging contrast agents in human serum at 37 degrees $\mathrm{C}$. Invest Radiol. 2008;43:817-828.

15. Frenzel T, Apte C, Jost G, et al. Quantification and assessment of the chemical form of residual gadolinium in the brain after repeated administration of gadoliniumbased contrast agents: comparative study in rats. Invest Radiol. 2017;52.

16. Runge VM. Safety of the gadolinium-based contrast agents for magnetic resonance imaging, focusing in part on their accumulation in the brain and especially the dentate nucleus. Invest Radiol. 2016;51:273-279.

17. Thomsen HS. T1 hyperintensity in the brain after multiple intravenous injections of gadolinium-based contrast agents. Acta Radiol. 2016;57:389-391.

18. Tweedle MF, Wedeking P, Kumar K. Biodistribution of radiolabeled, formulated gadopentetate, gadoteridol, gadoterate, and gadodiamide in mice and rats. Invest Radiol. 1995;30:372-380.

19. Bruder O, Schneider S, Pilz G, et al. 2015 Update on acute adverse reactions to gadolinium based contrast agents in cardiovascular MR. large multi-national and multi-ethnical population experience with 37788 patients from the EuroCMR registry. J Cardiovasc Magn Reson. 2015;17:58.

20. Morzycki A, Bhatia A, Murphy K. Adverse reactions to contrast material: a canadian update. Can Assoc Radiol J. 2016;pii: S0846-5371:30043-2.

21. Radbruch A. Are some agents less likely to deposit gadolinium in the brain? Magn Reson Imaging. 2016;34:1351-1354.

22. Semelka RC, Ramalho M, AlObaidy M, et al. Gadolinium in humans: a family of disorders. AJR Am J Roentgenol. 2016;207:229-233.

23. Burke LM, Ramalho M, AlObaidy M, et al. Self-reported gadolinium toxicity: a survey of patients with chronic symptoms. Magn Reson Imaging. 2016;34:1078-1080.

24. Welk B, McArthur E, Morrow SA, et al. Association between gadolinium contrast exposure and the risk of parkinsonism. JAMA. 2016;316:96-98.

25. Agarwal A, Kanekar S, Sabat S, et al. Metronidazole-induced cerebellar toxicity. Neurol Int. 2016;8:6365.

26. Murata N, Gonzalez-Cuyar LF, Murata K, et al. Macrocyclic and other non-group 1 gadolinium contrast agents deposit low levels of gadolinium in brain and bone tissue: preliminary results from 9 patients with normal renal function. Inves Radiol. 2016;51:447-453.

27. Lancelot E. Revisiting the pharmacokinetic profiles of gadolinium-based contrast agents: differences in long-term biodistribution and excretion. Invest Radiol. 2016;51:691-700.

28. Vidaud C, Bourgeois D, Meyer D. Bone as target organ for metals: the case of f-elements. Chem Res Toxicol. 2012;25:1161-1175.

29. Spasovski GB, Sikole A, Gelev S, et al. Evolution of bone and plasma concentration of lanthanum in dialysis patients before, during 1 year of treatment with lanthanum carbonate and after 2 years of follow-up. Nephrol Dial Transplant. 2006 21:2217-2224.

30. Shigematsu T. Lanthanum Carbonate Research Group. Three-year extension study of lanthanum carbonate therapy in Japanese hemodialysis patients. Clin Exp Nephrol. 2010;14:589-597.

31. Robert P, Lehericy S, Grand S, et al. T1-weighted hypersignal in the deep cerebellar nuclei after repeated administrations of gadolinium-based contrast agents in healthy rats: difference between linear and macrocyclic agents. Invest Radiol. 2015;50:473-480.

32. Robert P, Violas X, Grand S, et al. Linear gadolinium-based contrast agents are associated with brain gadolinium retention in healthy rats. Invest Radiol. 2016;51:73-82. 
33. Jost G, Lenhard DC, Sieber MA, et al. Signal increase on unenhanced T1weighted images in the rat brain after repeated, extended doses of gadoliniumbased contrast agents: comparison of linear and macrocyclic agents. Invest Radiol. 2016;51:83-89.

34. Rasschaert M, Idée JM, Robert P, et al. Moderate renal failure accentuates T1 signal enhancement in the deep cerebellar nuclei of gadodiamide-treated rats. Invest Radiol. 2017;52.

35. Smith AP, Marino M, Roberts J, et al. Clearance of gadolinium from the brain with no pathologic effect after repeated administration of gadodiamide in healthy rats: an analytical and histologic study. Radiology. 2017;282:743-751.

36. Schlemm L, Radbruch H, Brandt AU, et al. Histopathologic assessment of neurotoxicity after repeated administration of gadodiamide in healthy rats. Radiology. 2017;282:925-926.

37. Lohrke J, Frisk A, Frenzel T, et al. Histology and gadolinium distribution in the rodent brain after the administration of cumulative high doses of linear and macrocyclic gadolinium-based contrast agents. Invest Radiol. 2017;52.

38. Roberts DR, Welsh CA, LeBel DP 2nd, et al. Distribution map of gadolinium deposition within the cerebellum following GBCA administration. Neurology. 2017.

39. Giorgi H, Ammerman J, Briffaux JP, et al. Non-clinical safety assessment of gadoterate meglumine (Dotarem(®)) in neonatal and juvenile rats. Regul Toxicol Pharmacol. 2015;73:960-970.

40. Fretellier N, Maazouz M, Luseau A, et al. Safety profiles of gadolinium chelates in juvenile rats differ according to the risk of dissociation. Reprod Toxicol. 2014; 50:171-179.

41. Oner AY, Barutcu B, Aykol S, et al. Intrathecal contrast-enhanced magnetic resonance imaging-related brain signal changes: residual gadolinium deposition? Invest Radiol. 2017;52:195-197.
42. Jost G, Frenzel T, Lohrke J, et al. Penetration and distribution of gadolinium-based contrast agents into the cerebrospinal fluid in healthy rats: a potential pathway of entry into the brain tissue. Eur Radiol. 2016.

43. Lindner U, Lingott J, Richter S, et al. Analysis of gadolinium-based contrast agents in tap water with a new hydrophilic interaction chromatography (ZICcHILIC) hyphenated with inductively coupled plasma mass spectrometry. Anal Bioanal Chem. 2015;407:2415-2422.

44. Thomsen HS. Are the increasing amounts of gadolinium in surface and tap water dangerous? Acta Radiol. 2017;58:259-263.

45. Birka M, Roscher J, Holtkamp M, et al. Investigating the stability of gadolinium based contrast agents towards UV radiation. Water Res. 2016;91:244-250.

46. Cao Y, Zhang Y, Shih G, et al. Effect of renal function on gadolinium-related signal increases on unenhanced T1-weighted brain magnetic resonance imaging. Invest Radiol. 2016;51:677-682.

47. Zhang Y, Cao Y, Shih GL, et al. Extent of signal hyperintensity on unenhanced T1-weighted brain $\mathrm{mr}$ images after more than 35 administrations of linear gadolinium-based contrast agents. Radiology. 2017;282:516-525.

48. Cenci MA, Whishaw IQ, Schallert T. Animal models of neurological deficits: how relevant is the rat? Nat Rev Neurosci. 2002;3:574-579.

49. Shah A, Garzon-Muvdi T, Mahajan R, et al. Animal models of neurological disease. Adv Exp Med Biol. 2010;671:23-40.

50. Runge VM, Wells JW, Williams NM. Evaluation of gadolinium 2, 5-BPA-DO3A, a new macrocyclic hepatobiliary chelate, in normal liver and metastatic disease on high field magnetic resonance imaging. Invest Radiol. 1996;31:11-16.

51. Marinelli ER, Neubeck R, Song B, et al. Synthesis, characterization, and imaging performance of a new class of macrocyclic hepatobiliary MR contrast agents. Invest Radiol. 2000;35:8-24. 